\section{Does early androgen exposure contribute to autistic traits?}

By Dr. Jessica Edwards

Researchers in Hong Kong and Cambridge have explored the influence of early androgen exposure on autistic traits during childhood. It has been proposed that higher levels of androgens (such as testosterone) during early life might contribute to the development of autistic traits, and that this might explain why autism is more common in males than females. Karson Kung and colleagues collected data from 97 boys and 110 girls from the Cambridge Baby Growth Study. They measured the anogenital distance (AGD) and penile length - two putative biomarkers of early androgen exposure - at birth and at 3 months-of-age. Then they assessed autistic traits via a parent-reported questionnaire when the child reached 9-13 years-of-age. Consistent with previous studies, ${ }^{1,2}$ they found no significant associations between the two androgen exposure biomarkers and autistic traits in boys, girls or both sexes combined. Assuming that AGD and penile length are accurate biomarkers, it seems that early androgen exposure might not explain the sex bias observed in autism.
Referring to:

Kung, K. T. F. et al. (2020), No relationship between prenatal or early postnatal androgen exposure and autistic traits: evidence using anogenital distance and penile length measurements at birth and 3 months of age. J. Child Psychol. Psychiatr. doi: 10.1111/jcpp.13335.

\section{References:}

${ }^{1}$ Kung, K.T.F. et al. (2016). No relationship between early postnatal testosterone and autistic traits in 18 to 30-month-old children. Mol. Autism. 7, 15. doi: 10.1186/s13229-016-0078-8.

${ }^{2}$ Kung, K.T.F. et al. (2016). No relationship between prenatal androgen exposure and autistic traits: Convergent evidence from studies of children with congenital adrenal hyperplasia and of amniotic testosterone concentrations in typically-developing children. J. Child Psychol. Psychiatr. 57, 1455-1462. doi: 10.1111/jcpp.12602. 